ANUARIO DE Estudios MEdiEVALES 42/1, enero-junio de 2012, pp. 141-161

ISSN 0066-5061

doi:10.3989/aem.2012.42.1.07

\title{
PREDICAZIONE E CURA PASTORALE. I SERMONI DELLA CLARISSA VENEZIANA CHIARA BUGNI $(1471-1514)^{1}$
}

\author{
PREACHING AND PASTORAL CARE. \\ THE SERMONI OF THE VENETIAN CLARISSAN NUN CHIARA BUGNI \\ (1471-1514)
}

GABRIELLA ZARRI Università di Firenze

\begin{abstract}
Riassunto: Si analizzano i Sermoni della clarissa veneziana Chiara Bugni (14711514) a partire dalla recente edizione dal Libro della beata Chiara, composto alla metà del Cinquecento. I Sermoni documentano la prassi della predicazione dell'abbadessa all'interno del monastero e sono distinte dalle visioni e rivelazioni affidate alla Vita composta dal confessore. Si conferma così una duplice funzione della predicazione femminile: quella profetica, rivolta all'esterno, e quella ex officio di carattere esortativo e pastorale.
\end{abstract}

Parole chiave: scrittura monastica; biografia e rivelazione; predicazione femminile ex officio.

\begin{abstract}
This article analyzes the Sermons of the Venetian Clarissian nun Chiara Bugni (1471-1514) based on a recent edition of the Libro della beata Chiara, composed in the mid-sixteenth century. The sermons document the practice of the preaching of the abbess of the monastery and they are distinct from the visions and revelations affirmed in the Vita composed by her confessor. This confirms a double function of feminine preaching: that of the prophetess, oriented outward, and that of an ex officio kind of a hortatory and pastoral character.
\end{abstract}

Keywords: monastic writing; biography and revelation; extra-official women's preaching.

\section{SOMMARIO}

1. Premessa.-2. Il Libro della beata Chiara.- 3. Chiara Bugni e la Vita composta da Francesco Zorzi.- 4. La predicazione ex officio.- 5. Il testamento spirituale.- 6. I Sermoni di Chiara Bugni alle consorelle: il fondamento della carità.- 7. I Sermoni di Chiara Bugni alle consorelle: la pazienza e l'umiltà.- 8. I Sermoni di Chiara Bugni alle consorelle: l'ubbidienza.- 9. Il congedo di Chiara Bugni: la predicazione femminile.- 10. Bibliografia.

\footnotetext{
${ }^{1}$ Abbreviature utilizzate: ASV = Archivio di Stato di Venezia; Libro = Libro della beata Chiara.
} 


\section{PREMESSA}

Per secoli ignorata dall' Ordine religioso a cui si era consacrata, dimenticata perfino nell' ambiente cittadino in cui era vissuta, la clarissa Chiara Bugni è ritornata recentemente alla memoria come protagonista di una vicenda mistica e intellettuale che molto ha da dirci sulla religiosità del primo Cinquecento veneziano. Professa nel monastero del Santo Sepolcro di Venezia, fondato da diverse nobildonne sfuggite alla presa di Negroponte da parte dei Turchi nel 1470, visse nascostamente per un quindicennio fino a quando il confessore non rivelò i doni mistici di cui Chiara Bugni era stata insignita. Tra il 1507 circa e il 1511 tutta Venezia seguì con partecipazione ed interesse il gran mistero che si svolgeva tra le mura monastiche. L'eccessivo rumore provocò l'intervento di un visitatore dell'ordine francescano che impose alla clarissa il silenzio e la carcerazione nel monastero. Dopo tre anni la mistica morì lasciando dietro di sé una duratura fama di santità.

Gli studi sulla clarissa veneziana hanno ricevuto impulso nell'ultimo trentennio sulla scia dell'interesse per la dottrina e le opere del teologo francescano Francesco Zorzi, Guardiano del monastero di San Francesco della Vigna e direttore spirituale delle monache del Santo Sepolcro, e nell'ambito delle ricerche sulla santità femminile e sul fenomeno delle "sante vive" nel primo Cinquecento ${ }^{2}$. Da questo congiunto interesse sono scaturite indagini importanti tanto sul piano dell'approfondimento filosofico che documentario. La vita di Francesco Zorzi, teologo cabalista, autore di opere che ebbero fama europea nel secolo XVI e oltre, è stata puntualmente ricostruita da Saverio Campanini ${ }^{3}$ e la biografia di Chiara Bugni ha avuto una propria collocazione all'interno della storia complessiva della comunità monastica in cui era vissuta attraverso la pubblicazione del Libro della beata Chiara, opera che segna una tappa miliare nella conoscenza della vita religiosa del primo Cinquecento ${ }^{4}$.

\section{IL LIBRO DELLA BEATA CHIARA}

Prima della pubblicazione del Libro della beata Chiara, la vita e i sermoni di Chiara Bugni erano conosciuti tramite una edizione seicentesca ${ }^{5}$ e un testimone manoscritto conservato nel convento veneziano di San Francesco della Vigna ${ }^{6}$. A questi testi fanno riferimento gli studi fin qui prodotti e sopra citati. Il lungo lavoro di ricerca condotto in relazione all'edizione di questo manoscritto ha fatto emergere nuovi testimoni e in particolare ha portato alla luce il Libro della beata Chiara, confezionato nella seconda metà del Cinquecento dal confessore del monastero, il sacerdote fiorentino Andrea Pillolini, in collaborazione con le monache. Composto tra il 1562 e

${ }^{2}$ Si citano i primi saggi di riferimento su Francesco Zorzi e Chiara Bugni: C. Vasoli, Intorno a Francesco Giorgio Veneto, pp. 129-403. Per i rapporti con Chiara Bugni: idem, Un "precedente" della Vergine Veneziana, pp. 203-225; G. Zarri, Le sante vive, p. 96 e rispettive note; eadem, Madri dell'anima, vol. XVII, pp. 415-435.

${ }^{3}$ F. Zorzi, L'armonia del mondo, pp. 11-43; cf. anche G. Busi, Francesco Zorzi. Un metodico sognatore, pp. 161-186.

${ }^{4}$ L'edizione critica del Libro è in: R. Mueller, G. Zarri (eds.), La Vita $e$ i Sermoni di Chiara Bugni. Nelle pagine che seguono riporto alcune informazioni più ampiamente espresse nel saggio introduttivo a questa edizione: G. Zarri, Chiara Bugni e Francesco Zorzi suo biografo, pp. 11-39.

${ }_{5}^{5}$ Marco da Lisbona, Croniche de li Ordini instituiti, parte IV, tomo III.

${ }^{6}$ Questo manoscritto è stato oggetto della tesi di laurea di Stefania Cavalli, che ne ha condotto l'edizione. S. Cavalli, Beata Chiara Bugni. 
il 1563, il libro contiene la traduzione della vita di Chiara Bugni tratta dagli appunti latini di Francesco Zorzi, le Esortazioni, o sermoni, dati dalla mistica alle consorelle e trascritti da una monaca di nome Augustina, infine una seconda vita di Chiara Bugni compilata all'interno del monastero. Più completo rispetto agli altri testimoni e primo in ordine di tempo, il Libro della beata Chiara, ora conservato al Museo Correr di Venezia, appare anche più consono ad illustrare un peculiare esempio di scrittura monastica condotta da più persone e su diversi registri.

Basilare resta la vita del primo biografo, il colto Francesco Zorzi, che consegna un testo incompiuto ma già organizzato per essere completato con immagini e figure. L'andamento diaristico della biografia lascia supporre che essa fosse stata compilata durante la vita della Bugni o almeno poco tempo dopo la sua morte. Il progetto non fu tuttavia portato a compimento per il mutare degli interessi del teologo o forse per il mutare dei tempi. Certo la stesura di questo testo deve collocarsi in data anteriore al 1540, anno di morte dello Zorzi. I diversi decenni trascorsi tra questo primo resoconto delle visioni e miracoli della clarissa e la compilazione del Libro ora edito sono carichi di eventi sconvolgenti per la città di Venezia e per l'intera cristianità. Lo stesso biografo, noto per cultura e santità di vita nei primi decenni del Cinquecento, a metà secolo risulta ormai autore sospetto di eresia e i suoi testi maggiori saranno di lì a poco sottoposti a emendazione e posti all'indice ${ }^{7}$. Ciò non impedisce che all'interno del monastero egli goda ancora di grande rispetto e autorità e Andrea Pillolini ne traduce fedelmente lo scritto inserendolo in una compilazione che non ha soltanto uno scopo edificante, ma è probabilmente pensata in funzione di una auspicata canonizzazione di Chiara Bugni.

A glorificare la mistica clarissa concorrono dunque la fama in vita, la biografia di un teologo illustre, le parole della santa raccolte e trascritte dalle consorelle ed infine un nuovo ritratto elaborato all'interno del convento, mettendo a frutto le informazioni mancanti nella prima biografia e le virtù e miracoli della beata filtrati attraverso l'esperienza delle consorelle e tramandate nella seconda biografia.

Il Libro della beata Chiara appare dunque l'espressione più compiuta della continuità della fama di santità della Bugni all'interno del convento e della volontà di promuoverne il culto; e al tempo stesso rappresenta un esempio singolare di scrittura conventuale comunitaria in cui concorrono, con unità d'intenti e pari dignità, noti teologi, colti sacerdoti e monache acculturate.

In questo breve saggio non si potrà dar conto di tutti gli aspetti del Libro che presentano interesse dal punto di vista storico. Accenneremo alla vita scritta da Francesco Zorzi e approfondiremo maggiormente i Sermoni di Chiara Bugni, ma vorremmo ugualmente sottolineare la cornice in cui questi testi vengono inseriti.

Le estese ricerche condotte da Reinhold C. Mueller sull' ambiente e le figure che circondano Chiara Bugni forniscono nuova luce sull'origine del monastero veneziano del Santo Sepolcro e sulle prime fondatrici ${ }^{8}$. Esse confermano tuttavia le asserzioni con cui si aprono le prime pagine del Libro della beata Chiara: il racconto della fondazione del monastero. La vita della monaca illustre in santità non viene in questo scritto composito proposta e rappresentata come un unicum avulso dal contesto in cui opera: essa viene inserita nel tessuto vivente di una comunità che la eleva ad esempio di virtù e di dottrina presentandola tuttavia come la parte di un tutto. Nel redigere $I l$ libro della beata Chiara la comunità del Santo Sepolcro intende fare memoria della propria origine ed identità, con lo scopo di consegnare questa memoria alle consorelle che si affilieranno al convento.

\footnotetext{
${ }^{7}$ C. Vasoli, Nuovi documenti sulla condanna all'indice, pp. 55-78.

${ }^{8}$ R. Mueller, Ambienti ecclesiastici e laici, pp. 63-122.
} 
Come avviene anche in altri casi, e in particolare nei monasteri osservanti, a partire dalla metà del Quattrocento le monache acquistano maggiore coscienza della propria identità, contrassegnata da una più rigorosa disciplina, e intraprendono la stesura di cronache monastiche il cui fine è quello di fare memoria del proprio modus vivendi e delle religiose che vivono santamente ${ }^{9}$. Tra le cronache italiane più significative per continuità e ricchezza di particolari si segnalano il Memoriale di Monteluce, delle clarisse perugine ${ }^{10}$, e quello di Santa Lucia di Foligno, culla dell'osservanza francescana nell'Italia centrale ${ }^{11}$.

Non mancano cronache monastiche anche nell'ambiente veneziano. Tra queste spicca per originalità e per intento polemico quella del Monastero delle Vergini. Composta negli anni Venti del Cinquecento per rivendicare i privilegi delle nobili professe che si oppongono alla riforma imposta dal Patriarca Antonio Contarini, il testo è redatto in latino e corredato da ricche miniature. Diversa per scopi, dichiaratamente volti a contrastare vita comune e clausura, ma ugualmente diretta a trasmettere monumento della nobiltà del monastero e della cultura delle professe, la cronaca del monastero delle Vergini partecipa di un comune intento di lasciare memoria dell'istituzione e del suo peculiare modo di vivere ${ }^{12}$. Come diversi studi recenti hanno rilevato, la conservazione e trasmissione della memoria di un monastero nell'età rinascimentale non è solamente affidata al ricordo delle donne illustri per santità ma è accompagnato da un preciso riferimento all'istituzione e a quanto in essa appare peculiare. Nel Libro della beata Chiara il ricordo della vita santa di Chiara Bugni è strettamente connesso con la disciplina e l'osservanza del monastero. Alle Esortazioni, ossia Sermoni, della abbadessa morente sono affidate le parole che fondano l'edificio spirituale della comunità. Carità, umiltà e obbedienza sono le virtù che le professe debbono osservare per vivere in pace e nella grazia del Signore. Le esortazioni pronunciate dalla beata in punto di morte sono il lascito che le consorelle raccolgono dalle labbra di Chiara Bugni e che trasmettono in scritto a coloro che verranno negli anni a seguire. Non è presente nel testo il ricordo delle altre professe del Santo Sepolcro morte in concetto di santità. E' noto infatti che Beatrice Venier, una delle fondatrici del monastero, era ritenuta beata al pari della Bugni. La sua influenza spirituale non aveva tuttavia raggiunto la fama di Chiara, la cui vita miracolosa aveva attratto l'attenzione di principi, alti prelati e città. Il ricordo della Venier avrà ugualmente un posto di prestigio nella memoria del monastero, ma sarà destinato ad un luogo diverso: il Catastico delle possessioni del convento ${ }^{13}$. Finalizzato a lasciare testimonianza della fondazione e ampliamento dell'istituto conventuale, il libro del Catastico si apre infatti con la biografia della Venier, una delle prime professe che era convissuta con Chiara in unità d'intenti e santità di vita. Comprenderemo meglio i motivi della fama di santità di Chiara Bugni addentrandoci brevemente nell'analisi della vita della clarissa tramandataci da Francesco Zorzi, francescano dell'Osservanza e curatore spirituale del convento.

\footnotetext{
${ }^{9}$ Su queste tematiche véase da ultimo: G. Zarri, N. Baranda Leturio (eds.), Memoria e comunità femminili.

${ }^{10}$ U. Nicolini (ed.), Memoriale di Monteluce; G. Zarri, R. Chiacchella (coords.), Memoriale di Monteluce, vol. II.

${ }^{11}$ Ricordanze del Monastero di Santa Lucia.

${ }^{12}$ G. Zarri, Venetian Convents, pp. 37-56; K. Lowe, Nuns' Chronicles.

${ }_{13}$ ASV, Santo Sepolcro, b. 1, reg. A, Catastico dei Beni. Sulle fonti relative a Beatrice Venier si veda il puntuale intervento di S. Rauch, La Vita della beata Chiara Bugni, pp. 3-26.
} 


\section{ChiARA Bugni E LA VITA COMPOSTA DA FRANCESCO ZORZI}

La santità di Chiara Bugni appariva particolarmente significativa agli occhi dei contemporanei per la singolarità delle grazie che le erano state concesse e per la missione a cui si diceva chiamata in un alternarsi di visioni ed ostensioni di oggetti materiali di origine sacra che conferivano alla religiosa un segno inequivocabile di predilezione divina e di santità. Di nobile famiglia, Chiara nacque a Venezia nel 1471. Entrò nel 1489 nella comunità di terziarie francescane del Santo Sepolcro, fondata nel 1481, che divenne di clausura nel 1499, sotto la direzione dei Francescani Osservanti. Nel 1504 venne eletta abbadessa, carica che detenne fino al 1511, anno in cui il provinciale dell'ordine vietò la sua rielezione. Durante il suo ufficio si erano infatti manifestati alcuni dei più importanti prodigi che la riguardavano e un Commissario visitatore, inviato dai superiori dell'ordine nello stesso 1511 si dimostrò scettico nei confronti dei fenomeni ritenuti miracolosi e deprecò il singulare et peregrinum genus $v_{\text {vitae }}{ }^{14}$ della clarissa, obiettando che la quiete del convento era perturbata per l'eccessiva frequenza delle visite. A Chiara venne dunque imposto il silenzio e la carcerazione; fu costretta infatti a vivere in abdito domus recessu ab hominum Sororumque conspectu semotam ${ }^{15}$. Pochi anni dopo, nel giorno delle Stigmate di san Francesco, il 17 settembre 1514, Chiara Bugni morì lasciando nel dolore le consorelle che continuarono a prestare fede alla sua santità e al suo insegnamento. La vita di astinenza e le visioni della clarissa erano di fatto state divulgate in città fin dal 1507 e divennero presto note, tanto che lo stesso Marin Sanudo, senatore della Repubblica e storiografo, diede spesso ragguaglio nei suoi Diarii degli eventi che riguardavano la donna e il monastero ${ }^{16}$. Quanto sappiamo di lei, tuttavia, si deve quasi interamente alla leggenda agiografica composta da Francesco Zorzi.

Quali erano le rivelazioni della clarissa che tanto avevano interessato il colto biografo e avevano destato scalpore nell' ambiente cittadino? Quali potevano essere stati i motivi che avevano spinto Francesco Zorzi ad annotare quelle visioni e ad abbandonare successivamente il proposito di comporre una biografia della Bugni? Senza dubbio l'interesse del teologo ha una motivazione culturale e speculativa in ordine al problema allora scottante della profezia vera e falsa, intorno a cui si concentravano le riflessioni dei domenicani savonaroliani e di filosofi eclettici come Giovan Francesco Pico; e certo non poteva mancare il tentativo di interpretare in senso cabalistico le manifestazioni che riguardavano la mistica clarissa. Tuttavia i fenomeni mistici che concernevano Chiara Bugni erano strettamente connessi con temi e problemi, quali la stigmatizzazione e i miracoli eucaristici, che nei primi due decenni del Cinquecento attiravano l'attenzione dei teologi e dell" "intellighenzia" religiosa e laica di diversi paesi europei. Prima di indicare alcuni possibili spunti interpretativi concernenti i fenomeni mistici di Chiara Bugni, è opportuno esaminare il racconto che Francesco Zorzi ne fornisce con ricchezza di particolari.

Le prime visioni della clarissa veneziana riportate nella Vita del teologo francescano risalgono all'anno 1503 e si infittiscono tra il 1506 e il 1507, per poi diradarsi lentamente. Hanno il carattere di rivelazione iniziatica. Più volte Chiara asserisce di essere tenuta al segreto, anzi una volta afferma esplicitamente: Sappi, figliuolo mio, come li doni divini vengono con tal conditione, che essendo tenuti secreti,

\footnotetext{
${ }^{14}$ L. Wadding, Annales minorum, vol. VII, pp. 541-548: citazioni a p. 547.

15 Ibidem.

${ }^{16}$ Sulla vita e l'ambiente della monaca rinvio al saggio di R. Mueller, Ambienti ecclesiastici e laici, pp. 63-122.
} 
si conservano, et manifestati si perdono ${ }^{17}$. Nonostante ciò, la mistica rivela a poco a poco i doni che le sono stati dati, premurandosi di dichiarare di farlo con il consenso di Dio, fino a pretendere che siano pubblicati, cioè resi pubblici, dallo stesso Francesco Zorzi ${ }^{18}$.

In questo graduale processo di disvelamento, la Bugni alterna reticenze con manifestazioni di impazienza, che ricalcano le invettive profetiche di Geremia, come quando scrive lettere per ingiungere ai francescani di predicare l'annuncio della redenzione del mondo ${ }^{19}$. Quanto al carattere di segretezza, questo è mantenuto dallo stesso Zorzi e forse imposto dalle autorità ecclesiastiche, data la straordinarietà degli eventi e delle manifestazioni miracolose che coinvolgono la clarissa e il monastero.

Nella primavera del 1507 il Cardinal Domenico Grimani, Protettore dell'ordine francescano, è incaricato di compiere una visita al monastero del Santo Sepolcro per accertarsi della veridicità dei fatti che avvengono in quel luogo, e poco tempo dopo la visita i "misteri" concernenti la monaca vengono divulgati in città. Sotto la data 15 aprile 1507 Marin Sanudo sintetizza con precisione i doni celesti ricevuti da Chiara Bugni:

L'abbadessa dil Sepulcro, qual non manza, vive di comunion, à auto sangue, late, aqua di Cristo in una impoleta, sì che è santa. El cardinal Grimani, suo protetor, quando el fu qui a Venecia, fo nel monasterio per veder, sì come ho scripto di sopra ${ }^{20}$.

Dalla testimonianza del cronista apprendiamo dunque che suor Chiara vive in completa astinenza e si ciba solo di eucarestia; ha inoltre ricevuto dal cielo sangue e acqua di Cristo e latte della Vergine divise in diverse ampolle. Le annotazioni di Marin Sanudo sono l'unica testimonianza esterna alla biografia fino ad ora reperita. Per addentrarci nei misteri di Chiara occorre dunque affidarci alle parole di Francesco Zorzi.

Il teologo francescano compone in un tutto armonico l'esperienza mistica della donna, raccontando come dopo la conversione, avvenuta ascoltando una predica, Chiara entrò in monastero e qui iniziò una vita di austerità e penitenza, descritta secondo la tipologia trasmessa dalla leggenda agiografica di Caterina da Siena: la religiosa digiuna costantemente e vive solo di eucaristia, è unita in matrimonio con Cristo, è gratificata di visioni, ha spirito di profezia e fa miracoli.

Eletta abbadessa nel 1504, già in fama sanctitatis, la Bugni fu insignita dei doni più straordinari proprio nel periodo del suo ufficio. Compì alcune guarigioni servendosi anche di un fazzoletto bagnato nel "misterioso liquore" che le era stato inviato dal cielo ${ }^{21}$, ma è soprattutto in occasione della comunione che ella poté godere dei maggiori doni mistici. Quasi sempre dopo la comunione era soggetta ad estasi che duravano interi giorni. Quando non le era consentito ricevere il sacramento, invece, si scioglieva in lacrime. Nonostante avesse una profonda devozione all'eucarestia, era

\footnotetext{
${ }^{17}$ Si cita dal Libro edito in: R. Mueller, G. Zarri (eds.), La Vita e $i$ Sermoni di Chiara Bugni: Libro, f. 13 r, p. 155 .

18 "Sopra le tue spalle, fra Francesco, da Dio ti è imposto questo (...). Tu predicherai in piazza di San Marco. Quivi fa di bisogno che tu mostri questo celeste thesoro. Io te lo darò sopra la fede tua acciò che tu facci quello che ha ordinato l'altissimo Idio". Libro, f. 80r, p. 207.

19 Ibidem, ff. $111 \mathrm{r}-\mathrm{v}$, p. 231.

${ }^{20}$ M. Sanudo, I Diarii, VII, col. 42.

${ }^{21}$ Guarisce una consorella inviandole una mela da mangiare, guarisce anche il nipote di fra Francesco: Libro, ff. 15r-v, pp. 156-157.
} 
tentata sulla realtà della transustanziazione e un giorno le apparve il Bambino Gesù sopra la patena ${ }^{22}$.

Fino a questo punto la rappresentazione agiografica della clarissa veneziana non si discosta molto da quella di Caterina da Siena; si deve soltanto notare una maggiore rilevanza data alla devozione eucaristica, in conformità con la tradizione di Chiara d'Assisi e di altre sante clarisse del secolo XV, come Caterina de' Vigri ${ }^{23}$. Nel racconto dello Zorzi, tuttavia, si riscontrano alcune lacune proprio in corrispondenza dell'evento più clamoroso che riguarda direttamente la Bugni e che era stato divulgato dallo stesso Marin Sanudo: il dono da parte di Cristo di alcune gocce del suo sangue racchiuse in una ampolla. A questo proposito la leggenda contiene però la descrizione dell'estasi in cui Chiara Bugni riceve la rassicurazione da parte del Salvatore stesso che in quell'oggetto materiale trasmessole misteriosamente non vi è inganno, ma che quel sangue le è stato dato per la remissione dei peccati di coloro che avranno fede. Nella medesima visione vi è anche l'avvertimento di Cristo che per quel dono la religiosa è destinata a patire fino alla morte ${ }^{24}$.

Siamo dunque in presenza di una imitatio Christi completamente realizzata da parte della mistica, che è chiamata per questo a una "morte vicaria", in sostituzione e ripetizione di quella di Cristo stesso. Di lì a poco infatti ci verrà detto dall'agiografo che Chiara ha una piaga nel costato che sanguina.

Il testo della precedente visione contiene anche alcuni elementi fondamentali per comprendere la missione di Chiara Bugni. Come nuova Maria Maddalena, la vergine veneziana ha il compito di annunciare per prima al mondo la misericordia di Dio; tale misericordia si manifesterà nella sofferenza di Chiara per la salvezza della umanità. Ella sarà tramite della illuminazione del mondo per il sangue che le è stato donato: il mondo sarà illuminato per questo sangue $e^{25}$.

Una serie di fatti miracolosi si sussegue con rapidità intorno al sangue ricevuto dalla Bugni dal cielo. Esso bolle nell'ampolla e aumenta di volume; nel sangue si deposita un liquore che viene posto in più ampolle custodite in un oratorio; un giorno, destatasi da un'estasi, la clarissa corre all'oratorio e vede le ampolle sparse d'un qualche sudore di latte et quel liquore se n'era ridotto al fondo ${ }^{26}$; qualche tempo dopo ancora, il liquore di balsamo sale alla superficie del sangue; nel mezzo dell'ampolla si forma uno splendore in forma di croce e Chiara e le sorelle vedono alcune gocciole che uscivano dal collo della ampolla ${ }^{27}$.

A questo punto il significato simbolico del sangue di Cristo che aumenta di volume, che produce balsamo e latte, da cui si distaccano alcune gocciole per uscire dal vaso di ampolla -simbolismo che rinvia tanto ai sacramenti dell'Unctio battesimale, crismale e sacerdotale, quanto al fatto biologico della generazione- si mescola al significato del sangue che esce dal costato di Chiara. La piaga laterale della mistica è infatti esaminata dai confessori, il suo sangue è rosso e profumato, non coagula, guarisce dalle malattie, anch'esso è raccolto in una ampolla ${ }^{28}$. Ora Chiara è diventata non solo un alter Christus, ma anche un secondo san Francesco, come lui insignita del dono delle stigmate.

Non procederò oltre nella descrizione dei fenomeni mistici di Chiara per la cui esposizione e interpretazione rinvio al saggio introduttivo citato, vorrei soltanto

22 Libro, f. 24v, p. 164.

${ }^{23}$ Tra i molti studi recenti, v. C. Leonardi (ed.), Caterina Vigri.

${ }^{24}$ Libro, f. 26r-v, pp. 165-166.

${ }^{25}$ Ibidem, ff. $26 \mathrm{v}-27 \mathrm{v}$, p. 166.

${ }^{26}$ Ibidem, f. $32 \mathrm{v}$, p. 171.

${ }^{27}$ Ibidem, f. $35 \mathrm{v}$, p. 173.

${ }^{28}$ Ibidem, ff. 41r-43r, pp. 177-178. 
ricordare che la Vita di Chiara Bugni composta da Francesco Zorzi elegge la monaca veneziana a testimone esemplare della ormai centenaria discussione teologica sul significato del sangue fluente e delle stigmate, riportata alla luce da un pregnante saggio di Caroline Bynum ${ }^{29}$; discussione rivitalizzata nel primo decennio del Cinquecento dal caso controverso delle stigmate di Lucia da Narni, terziaria domenicana protetta dal duca Ercole I d'Este ${ }^{30}$.

Le trasformazioni che il sangue di Cristo subisce nelle ampolle dove Chiara lo aveva riposto e l'ulteriore dono fatto alla monaca del latte della Vergine Maria completano il discorso teologico relativo al sanguis Christi collegandolo alle conoscenze scientifiche del tempo relative alla generazione. Poiché si credeva che il corpo umano fosse generato dal sangue di donna, Giovanni da Capistrano argomentava che il corpo di Cristo fosse stato formato dal puro sangue mestruale del grembo di sua madre: ciò implicava dunque la conferma della dottrina dell'Immacolata Concezione. Come affermavano anche altri teologi, il pretium della redenzione era il sangue uterino di Maria. L'idea, tratta dalla teoria fisiologica, che il feto fosse formato dal sangue femminile divenne nella spiritualità del tardo medioevo una comune immagine di salvezza ${ }^{31}$.

Anche nello specifico aspetto della connessione tra sangue e generazione, che accompagnava il dibattito teologico medievale sul sangue di Cristo, possiamo riconoscere molti dei fenomeni attribuiti alla Vergine veneziana. Certamente i misteri di Chiara Bugni presentati da Francesco Zorzi in forma di visione avevano una forte valenza spirituale, ma toccavano anche dottrine su cui occorreva esercitare cautela e discernimento. Comprenderemo allora meglio di quanto si sia fatto fino ad ora il noto episodio in cui fu implicato un predicatore vicino a Chiara Bugni e Francesco Zorzi: il canonico lateranense Pietro da Lucca che nel 1511 venne sottoposto a una processo di fronte a diversi prelati e teologi per aver predicato a Mantova che Cristo era stato concepito nel cuore e non nel grembo della Vergine Maria. Accusato di predicare false dottrine, egli si giustificò dicendo che aveva sostenuto quella tesi come semplice opinione pia e devota, avendola appresa da una donna ritenuta santa ${ }^{32}$. Sarà forse stato anche il clamore suscitato da questo evento mantovano che avrà influito sulla decisione dei superiori dell'ordine francescano di intervenire disciplinarmente contro Chiara Bugni? La data del processo contro Pietro da Lucca e quella della visita del Commissario francescano al monastero del Santo Sepolcro coincidono: oltre non è possibile andare.

Certo occorrerà ricordare che alla stessa data anche a Bologna una pia donna guidata spiritualmente da Pietro da Lucca professava dottrine e manifestava fenomeni mistici analoghi a quelli di Chiara Bugni: si trattava di Elena Duglioli dall'Olio su cui ci siamo in altre occasioni soffermati ${ }^{33}$.

Tutti i misteri di Chiara Bugni possono essere interpretati come episodi che rientrano a pieno titolo nel dibattito teologico qui sommariamente ricordato e vengono presentati a conferma e difesa della piena unità di umanità e di divinità del sangue di Cristo donato dal cielo alla Vergine veneziana, finalizzato ad essere esposto alla adorazione dei fedeli per procurare loro misericordia e salvezza.

Se l'aspetto teologico e devozionale che abbiamo sopra esposto costituisce il tessuto connettivo che consente di dare spiegazioni razionali alle visioni e ai molti

${ }^{29}$ C. Walker Bynum, Wonderful blood.

${ }^{30}$ Cf. A. Matter, G. Zarri, Una mistica contestata.

${ }^{31}$ C. Walker Bynum, Wonderful blood, pp. 158-159.

${ }^{32} \mathrm{Il}$ primo a segnalare l'episodio è stato D. Cantimori, Le idee religiose del Cinquecento, pp. 7-53.

${ }^{33}$ G. Zarri, Le sante vive, pp. 165-196. 
misteri presenti nella Vita di Chiara Bugni composta da Francesco Zorzi, che cosa rimane delle precedenti interpretazioni che rinviavano quasi esclusivamente alla cultura ermetica e cabalistica del francescano veneziano? Molto probabilmente la Vita si dovrà interpretare usando diverse chiavi di lettura. Pur considerando che l'interpretazione teologica relativa al sanguis Christi appare ora convenientemente esplicativa, resto nella convinzione che Francesco Zorzi avesse identificato in Chiara Bugni la Madre di una rigenerazione universale e di una Concordia mundi che nel secondo decennio del Cinquecento, per i rapidi mutamenti politici e culturali, era destinata ad allontanarsi sempre più ${ }^{34}$.

\section{LA PREDICAZIONE EX OFFICIO}

Le Esortazioni di Chiara Bugni trascritte da suor Augustina e inserite nel Libro della beata Chiara ci introducono alla duplice tematica storiografica della scrittura femminile e della predicazione delle donne, su cui ho avuto occasione di soffermarmi in studi precedenti ${ }^{35}$, ma possono anche offrire nuova documentazione alla pratica del sermo monasticus nelle istituzioni religiose medievali e rinascimentali. Un'analisi dei Sermoni ci consente di esaminare dall'interno la cultura delle clarisse e di saggiarne tanto la formazione umanistica e letteraria quanto la profondità scritturistica e teologica; ci autorizza inoltre a ribadire la netta distinzione, sostenuta nei saggi sopra citati, tra annuncio di tipo profetico e predicazione ex officio, intendendo con questa espressione l'insegnamento impartito dalla Abbadessa, in virtù del proprio ufficio, all'interno delle mura monastiche.

Molte delle rivelazioni di Chiara Bugni raccolte e trascritte dal confessore fra Francesco Zorzi erano all'insegna della passione di Cristo e della croce. Come in altri famosi casi della mistica italiana o straniera, tra Chiara e Francesco si era instaurato un rapporto particolare: solo lui era in grado di "notare" le rivelazioni della donna; in sua assenza queste venivano disperse ${ }^{36}$.

Nel complesso degli scritti rimasti, e in particolare nel Libro della beata Chiara, si può riscontrare una netta distinzione tra rivelazioni e sermoni. Le prime sono tutte annotate nella vita scritta dallo Zorzi e ai secondi è riservato uno spazio a parte, essendo stati riportati di seguito alle pagine che contengono vita e rivelazioni. Questa netta ripartizione tra visioni, che contengono annunci profetici e verità rivelate, e esortazioni, che impartiscono insegnamenti alla comunità monastica, ha lo scopo di differenziare la qualità e la funzione della predicazione femminile, riservando l'annuncio profetico ai fedeli e discepoli esterni al monastero e indirizzando i sermoni all'interno del chiostro ${ }^{37}$.

Ciò che accomuna i sermoni di Chiara Bugni con quelli della più antica consorella Caterina de' Vigri è il fatto che siano stati dati in punto di morte ${ }^{38}$. Quando

${ }^{34}$ Su Chiara Bugni come Madre della rigenerazione universale della chiesa rinvio al saggio di chi scrive: Madri dell'anima: Chiara Bugni, Elena Duglioli e la rigenerazione della chiesa, che utilizza come fonte il manoscritto di San Francesco della Vigna trascritto nella tesi di Cavalli, ora superato dalla edizione critica del Libro della beata Chiara.

${ }^{35}$ G. Zarri, Predicatrici e madri spirituali, pp. 159-177; eadem, Places and Gestures of Women's Preaching, pp. 177-193. Anche questi due saggi utilizzano come fonte la trascrizione di S. Cavalli.

${ }^{36}$ Tra i molti studi, si veda: G. Barone, J. Dalarun (eds.), Angèle de Foligno.

${ }^{37}$ Ho approfondito questo tema nel saggio: G. Zarri, Predicatrici e madri spirituali.

${ }^{38}$ Ho sviluppato il confronto nel saggio ibidem (pp. 165-167). Nel recensire gli autografi e gli scritti di Caterina Vigri, Silvia Serventi accetta come autentici i sermoni di Caterina pervenuti in 
la monaca veneziana si ammala, infatti, fa chiamare le sorelle e dà loro una serie di esortazioni che paiono costituire una regola di vita. Tanto in Chiara che nella bolognese Caterina de' Vigri c'era forse il ricordo e l'esempio di san Francesco, che in punto di morte volle lasciare il suo testamento spirituale. Nel caso della Bugni, si può parlare infatti di un particolare lascito della madre abbadessa morente: una sorta di ordinamento della comunità disegnato sulla base di una riflessione sui voti e le virtù essenziali per conservare l'osservanza della regola monastica. Occorre infatti notare che i Sermoni della clarissa veneziana furono dettati in un periodo in cui le Costituzioni della comunità di recente fondazione erano in via di elaborazione. Esse vennero infatti approvate nel 1517, dopo la morte di Chiara Bugni.

In questa breve analisi non esaminerò le Esortazioni dal punto di vista della scrittura femminile; intendo invece situare il testo all'interno della discussione sulla predicazione delle donne nell'età medievale e rinascimentale ${ }^{39}$, con particolare riferimento alla tradizione del sermo monasticus. Per quanto attiene l'aspetto letterario, mi limiterò ad osservare che i Sermoni di Chiara Bugni mostrano una approfondita padronanza della Sacra Scrittura e una non superficiale conoscenza dei Padri della Chiesa.

Oltre alle citazioni esplicite dei Vangeli e delle lettere apostoliche, dei salmi e di altri libri dell'antico testamento, le Esortazioni dell'abbadessa del Santo Sepolcro alle consorelle sono intessute di riferimenti impliciti che costituiscono il tessuto connettivo dei diversi discorsi. Tra le autorità più frequentemente citate figurano i principali Padri e dottori della Chiesa, come Agostino, Ambrogio, Giovanni Crisostomo, Gregorio e Cassiodoro, ma sono presenti anche i fondatori degli ordini religiosi, con il richiamo alle regole di Benedetto e Francesco. Un posto privilegiato nelle citazioni occupano l'agostiniano Girolamo da Siena e la clarissa Caterina Vigri, ma non mancano riferimenti diretti alle sequenze e agli inni liturgici o i richiami a versi danteschi e a proverbi popolari ${ }^{40}$.

In sintonia con una buona conoscenza dei testi sacri e degli autori cristiani, anche la forma letteraria delle Esortazioni di Chiara Bugni presenta aspetti pregevoli. Colpisce particolarmente una specifica attenzione all'andamento retorico del discorso, in gran parte basato sulla ripetizione della parola chiave che costituisce il thema del sermone. In analogia con il noto passo della lettera paolina contenente l'elogio della carità, la clarissa veneziana fonda l'efficacia retorica del suo discorso sul progressivo ampliamento del concetto che intende sottolineare partendo sempre dalla iterazione della parola chiave. Si veda ad esempio come Chiara sviluppa il thema della pazienza tessendo l'elogio della "tribulatione":

La tribulatione è una bevanda salutifera et un'herba molto buona, più di tutte l'herbe del Paradiso. La castiga il corpo, il qual nondimeno in breve tempo si ha da risolvere in putredine, et rinfresca l'anima molto più nobile et che sempre ha da vivere. La tribulatione è una verga piena d'amore et paterna castigatione de' miei eletti. La qual cognoscendo, il mio profeta David disse: "La tua verga et il tuo bastone m'hanno consolato" (cfr. Sal. 23, 4). La tribulatione tira et spigne a Dio la persona, o

trascrizione seicentesca e recentemente pubblicati. Cf. C. Vigri, Laudi, trattati e lettere; C. Vigri, I Sermoni.

39 Tra gli studi più importanti: B. Mayne Kienzle, P. Walker Berkeley (eds.), Women Preachers and Prophets; J.A. Kay McNamara, Sisters in Arms; K.L. Jansen, The Making of the Magdalen; B. Roest, Predicazione femminile, pp. 119-154.

${ }^{40}$ Ringrazio Silvia Serventi per la lettura dei Sermoni di Chiara Bugni e per le competenti osservazioni e suggerimenti. 
voglia o no, et chi non ha provato le tribulationi et le tentationi, ditemi di gratia, che cosa sa costui? ${ }^{41}$

\begin{abstract}
Altrettanto avviene per l'elogio della carità:
La charità fraterna è un ligame di pace, la qual sola nutrisce la concordia nella sua habitacione et conserva l'unità christiana fra le sorelle. La charità è la madre della mansuetudine et madre generosa della pietà chatolica. La charità fraterna è spaventevole a' demoni, perché la fa vittoriose tutte quelle delle quali abbraccia la camera del cuore. La charità spegne l'incendio della libidine et cuopre ogni multitudine di peccati. La charità fa che la monaca sia molto chara a Dio et la fa compagna de' santi angeli. La charità conserva la Chiesa nella unità de' santi, fuor della quale nessuna conseguisce la salute eterna. La charità fraterna compone i costumi di quelle che stanno ne' monasteri et fa che Idio habita nel mezzo delle monache. La charità non fa dispiacer ad alcuna, ma a tutte porge aiuto per compassione. La charità scaccia ogni vitio, perché ell’è sola nutrice di tutte le virtù. La charità conserva la reformatione della fraternità, nel vigore di quelle che stanno ne' monasteri, et opera una gran salute dell'anime. La mutua charità conferma l'ubbidienza et fa la correttione fraterna fruttuosa. La charità nutrisce la vera concordia fra le sorelle et conserva la castità bene odorifera, così delle menti come de' corpi. La charità fa che le monache, nella casa di Dio, siano d'un medesimo animo et in ogni buona opera lodevolmente concorde ${ }^{42}$.
\end{abstract}

La formula iterativa del thema si ripete anche nei sermoni sulla umiltà e l'ubbidienza, costituendo una caratteristica peculiare della scrittura di Chiara Bugni che assume una dignità letteraria superiore a quella di altre scrittrici coeve. Per quanto attiene le modalità espressive della scrittura si può notare ancora l'uso frequente delle forme dialogiche, tipiche della civiltà della "conversazione", qui adoperate anche in stretta connessione con il concetto di "conversatio" monastica.

\title{
5. IL TESTAMENTO SPIRITUALE
}

Nel Libro della beata Chiara le Esortazioni dell'abbadessa sono poste di seguito alla biografia incompiuta di fra Francesco Zorzi e sono inserite all'interno del racconto degli ultimi anni di vita della mistica. La parte del manoscritto che riproduce i sermoni inizia con un capitolo dal titolo: Questo che segue è della morte della beata Chiara $^{43}$. E' proprio in questo capitolo che apprendiamo della visita di un Commissario nel monastero del Santo Sepolcro e della proibizione dei superiori dell'Ordine francescano di rieleggere abbadessa Chiara Bugni dopo il settennato già espletato. Conosciamo inoltre la motivazione addotta per l'introduzione dei provvedimenti restrittivi nei confronti della mistica visionaria: il commissario rimprovera Chiara Bugni di questo suo voler vivere a suo modo e le ingiunge di rinchiudersi dentro il monastero ché tu non habbi a conversar con l'altre, et quivi vivi a tuo modo et fa le tue discipline et i tuoi digiuni; o veramente provediti d'andare ad habitar fuori di questo luogo ${ }^{44}$.

${ }^{41}$ Libro, f. 128r, p. 245.

${ }^{42}$ Ibidem, ff. 135v-136r, p. 251.

${ }^{43}$ Ibidem, f. 125r, p. 243.

${ }^{44}$ Ibidem. 
Secondo la testimonianza del Libro della beata Chiara, l'imposizione del carcere comminato alla abbadessa venerata nel monastero e nell'intera città appare determinata da motivi disciplinari, specialmente dallo scarso rispetto per la regola francescana. Non si fa menzione delle grazie mistiche e delle rivelazioni lasciate trapelare dai confessori e contenute nella Vita di Francesco Zorzi che avrebbero potuto essere considerate sospette dal punto di vista dottrinale. E' certo comunque che la monaca trascorse nel silenzio gli ultimi anni della vita accettando con spirito di rassegnazione la condanna imposta, considerata come una prova. E' in questa occasione che Chiara tesse l'elogio della pazienza e introduce una pregnante riflessione sulla tribolazione come martirio e via di salvezza:

La tribulatione è una via stretta, la qual fortemente perviene per insino a esse porte del cielo. Essa porta la persona alla compagnia de' martiri, essa riporta la laude et la palma di tutti gl' inimici, essa veste l'anima d'una veste di rosado et di purpura. Essa fa le ghirlande, over le corone di rose, et fa li scettri di palme verdi ${ }^{45}$.

Relegata in un luogo separato del monastero e privata del contatto con le altre sorelle, suor Chiara trascorse tre anni in penitenza, senza poter comunicare le rivelazioni che continuava a ricevere dal cielo a causa dell'assenza del confessore fra Francesco Zorzi:

Et così gli fu consegnato un luogo secondo il suo volere, dove quivi si fece un letticello di tavole con una stuora sotto et con un zocco di legno per capezzale. Et in quel luogo stette rinchiusa circa dua anni, dove hebbe di bellissime revelationi, le quali, per non esser stato fra /130r/ Francesco Giorgi nella città, non sono state notate. Et veramente essa poteva dire con san Paulo: "Vidi et audivi arcana Dei que non licet homini loqui" (1 Cor. 2, 9)

Secondo la testimonianza del Libro, durante il periodo di prigionia le rivelazioni della clarissa cessarono e lasciarono il posto alla predicazione che ella cominciò ad impartire in punto di morte. Le Exortationi sono il lascito spirituale della mistica, che agli occhi delle consorelle riveste ancore l'ufficio di abbadessa, e le sue parole costituiscono un insegnamento per vivere santamente in comunità e per raggiungere la salvezza:

Sorelle mie dilettissime, sentendomi horamai mancare tutti gli spiriti et cognoscendo che egli è venuta la fine della vita mia, che poco debba star con voi et che il mio Signore mi vuol cavar di queste tenebre et condurmi alla sua luce inaccessibile, amandovi come mie proprie figliole, vi ho fatto chiamare per ricordar alle charità di vostre alcune cose molto necessarie, le quali, se osserverete et farete il debito vostro, et vi farete degne di vita eterna. Et quel medesimo precetto ricorderò a voi, che dette il nostro Signore a' suoi discepoli quando disse: "Hoc est perceptum meum, ut diligatis invicem, sicut dilexi vos" (Gv. 13, 34)

L'esordio solenne della mistica morente, che richiama il primo precetto evangelico, si completa immediatamente con il riferimento alla lettera paolina che tesse l'elogio della carità e si sviluppa in un incalzante susseguirsi di esortazioni all'amo-

${ }^{45}$ Ibidem, f. 129r, p. 246.

${ }^{46}$ Ibidem, ff. 129v-130r, p. 246.

${ }^{47}$ Ibidem, f. 130v, p. 247. 
re reciproco. Nella prima esortazione e in tutte quelle successive il thema del Sermone è direttamente rapportato alla vita monastica, così che si può legittimamente affermare che la predicazione di Chiara Bugni è indirizzata esclusivamente alle consorelle e costituisce un insegnamento impartito ex officio. Ad un pubblico più vasto e non solo cittadino erano invece rivolte le visioni e rivelazioni che costituivano una sorta di predicazione profetica mediata attraverso la parola e la scrittura del confessore.

\section{I SERMONI DI CHIARA BUGNI ALLE CONSORELLE: IL FONDAMENTO DELLA CARITÀ}

La carità è il fondamento della vita monastica. Nel suo primo Sermone Chiara Bugni propone la differenza tra amore e carità e parla a lungo della carità tra le monache, che sono invitate a comportarsi come "ministre di Dio". La monaca non deve amare $i$ prossimi per affetto di parentado et di carne, come fanno i pagani, ma ama così il nimico come l'amico ${ }^{48}$. Questa prima affermazione, seguita dalla constatazione che vi sono molti che amano malamente perché vogliono tanto bene a qualcuno che perdono l'amor di Dio ${ }^{49}$, mostra in modo esplicito che la beata Chiara basa il proprio insegnamento sulla esperienza di Madre abbadessa e che i suoi sermoni hanno il fine di promuovere la concordia nel monastero, spesso turbato da lotte di fazione o di "partiti" tra le monache ${ }^{50}$.

Altro esempio di amore sbagliato è quello eccessivo o quello "particolare". Il primo è stolto, il secondo pericoloso. Le monache non debbono amare impudicamente e lascivamente perché nulla giova la verginità corporale se non vi è quella mentale $^{51}$. Anche l'invidia si oppone alla carità ed è espressione di amore di sé. Parentado, amicizie particolari, invidie e mormorazioni sono le cause più frequenti di discordia tra le monache. Solo la pratica della carità fraterna consente il superamento di questi ostacoli.

Il quadro della vita monastica che emerge indirettamente dal sermone di Chiara Bugni sulla carità mette a fuoco aspetti solitamente taciuti dalle fonti interne del monastero, che tendono a porre in evidenza la nobiltà e la santità della istituzione, e aprono uno spiraglio sui rapporti tra le monache e la loro vita di relazione. Particolarmente significative appaiono le diverse declinazioni della carità fraterna: la carità è un legame di pace, è madre della mansuetudine, spegne l'incendio della libidine ${ }^{52}$, fa che la monaca sia cara a Dio, conserva la chiesa nella unità, ha compassione di tutti, scaccia ogni vizio e nutre la virtù, conserva la reformatione della fraternità, scaccia l'odio e l'invidia, è ministra della giustizia e conserva la disciplina regolare nel monastero, conserva la mente pura, non biasima la povertà, non cerca i diletti della carne, ama la purità, non giudica, ma corregge umilmente il prossimo. In sintesi si può affermare che:

La charità fraterna è adempimento della legge, perché tutto quello che ci vien comandato, dopo il culto di Dio, si refferisce alla dilettione del prossimo. Di qui il santo amator di Christo disse: "Quello che ama il prossimo ha adempiuto la legge" 53 .

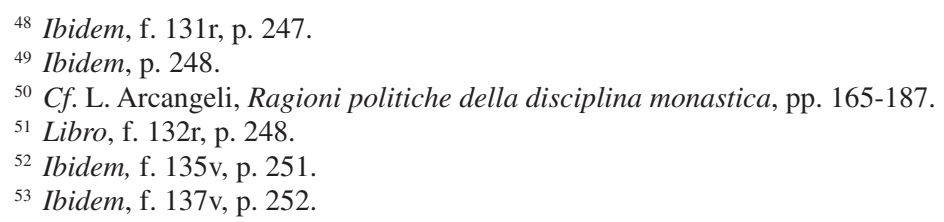


Dopo aver enunciato brevemente le caratteristiche principali della carità fraterna, la santa monaca riprende le sue affermazioni soffermandosi su alcuni aspetti particolari, come quello della amicizia delle monache e della concordia; ricorda infine che non gioverà alla salute praticare astinenze e rinunciare ai patrimoni familiari se non si consegue la carità fraterna.

Come il primo, il secondo e il terzo sermone hanno l'obiettivo di indicare le virtù che sono alla base della vita spirituale, ma anche di una serena convivenza tra le monache: la pazienza e l'umiltà. Queste virtù rinviano infatti ai fondamenti dell'imitazione di Cristo, e al tempo stesso favoriscono la reciproca sopportazione e comprensione.

\section{I SERMONI DI CHIARA BUGNI ALLE CONSORELLE: LA PAZIENZA E L’UMILTÀ}

Il sermone sulla pazienza è impartito da Chiara alle sorelle il giorno dopo aver pronunciato quello sulla carità. La condizione di debolezza fisica in cui si trova la mistica fa sì che ella debba riposarsi dopo aver sostenuto una lunga conversazione. Lo confessa la monaca stessa: Et havendovi assai sufficientemente parlato di questa santa charità, piglierò licenza dalle charitadi vostre di riposarmi alquanto, per sentirmi un poco stracca ${ }^{54}$. Come in questo caso, anche successivamente, l'insegnamento di Chiara Bugni viene impartito in giorni diversi, quasi a prolungare la conversazione tra sorelle e allontanare il momento del distacco.

Il sermone sulla pazienza si sviluppa dalla constatazione che questa virtù è particolarmente necessaria alle religiose che, come dice san Paolo, siamo fatte uno spettacolo a tutti gli huomini (1 Corinti 4,9) ${ }^{55}$. A proposito del richiamo paolino, si osserverà in premessa che qui e in tutti i Sermoni la citazione biblica è resa al femminile: segno evidente che l'enfasi del discorso è rivolto all'uditorio specifico delle monache a cui la predicazione è indirizzata. Si dovrà rilevare inoltre che la clarissa veneziana è attenta a contestualizzare i suoi discorsi, non soltanto sotto il profilo del pubblico, ma anche del clima culturale del tempo. Ella sa bene che al suo tempo la vita religiosa gode di scarsa considerazione e che le suore vengono beffeggiate, così da dover ricorrere alla paziente sopportazione. Del resto lo stesso Cristo Salvatore fu beffeggiato e deriso e può fornire l'esempio più significativo della virtù della pazienza. Questa si manifesta prima di tutto nella mancanza di superbia e nell'amore della povertà.

Dopo aver proceduto ad alcune esemplificazioni, Chiara Bugni riassume il suo insegnamento indicando una vera e propria classificazione della virtù della pazienza. Il primo grado è quello di coloro che non cercano volontariamente l'avversità e che si rattristano quando questa si presenti loro, però dopo una prima ribellione la sopportano pazientemente. Il secondo grado è di coloro che, pur non cercando di patir mali, sopportano il male come dono mandato da Dio. Il terzo grado della pazienza è il più perfetto e comprende quelle monache che si offrono spontaneamente alla derisione e alle avversità: come accade per il martirio, anche la virtù della pazienza può essere di due specie, a seconda che l'avversità sia ricercata o accettata.

Come i Martiri, anche i Confessori che non hanno subito la morte ma accettato la persecuzione possono costituire un esempio da imitare.

Terminata l'esortazione alla penitenza Chiara Bugni congeda le sue consorelle e le invita ad ascoltare il giorno seguente la predica sulla umiltà, virtù necessaria

${ }^{54}$ Ibidem, f. 142v, p. 256.

${ }^{55}$ Ibidem, f. 143r, p. 256. 
quanto la pazienza a quelle monache che veramente vogliono vivere in santa pace nel monastero ${ }^{56}$.

L'umiltà può essere considerata fondamento stabile di tutte le virtù, perché riconosce la condizione di peccato in cui la creatura è caduta, e continua a cadere facilmente, e non si insuperbisce. Con espressione icastica Chiara Bugni definisce l'umiltà come la "scala del cielo"; essa è tanto necessaria alla professione monastica che senza questa virtù non ci si può salvare. La santa abbadessa conduce questa riflessione applicandola alla realtà culturale e sociale dei monasteri rinascimentali, abitati per lo più da professe nobili, fiere dall'appartenenza a casati illustri:

La monaca gonfiata d'animo et superba, non è serva di Christo, ma del diavolo et non harà parte con Dio in cielo, ma co' demoni nell'inferno senza fine sarà tormentata. Imperò che Christo, maestro et precettor dell'humiltà, detesta et maledisce tutti li superbi, et non riceve in sua compagnia se non gli humili, miti et mansueti ${ }^{57}$.

Sulla contrapposizione del binomio umiltà-superbia si sviluppa successivamente il sermone di Chiara Bugni, che si conclude con la visione del giudizio e il premio riservato agli umili:

L'humiltà in cielo senza fine sarà coronata et la superbia patirà nell'inferno, in perpetuo, un cruciato inestimabile co' dimoni. L'humiltà coronata con Christo regnerà in cielo et la superbia, dispregiata, sarà cruciata nelle pene col diavolo ${ }^{58}$.

In questo contesto i superbi condannati al fuoco eterno comprenderanno l'errore fatto nel beffeggiare le umili monache durante la loro vita e dovranno riconoscere che le religiose hanno scelto la parte migliore:

Et vedendo i superbi et arroganti condennati all'inferno tanta gloria delle persone humili, le quali esse dispregiorno nel mondo, si maraviglieranno in tanta subitatione de' santi, et si turberanno con uno spaventevole timore, per l'angustia dello spirito gemendo et dicendo nel furor della superbia: "Queste son quelle monache povere et humili, già vestite di panni vili, separate dalle maritate, astenendosi dal mangiar carne et da ogni spurcitia di carne, le quali qualche volta noi le dileggiassimo et se ne ridavamo noi insensati. Noi pensavamo che la fine loro fusse una pazzia et la fine loro fusse senza honore. Ecco come hora sono computate, fra $\mathrm{i}$ figliuoli di Dio, et la loro sorte è fra li santi” ${ }^{59}$.

\section{I SERMONI DI CHIARA BUGNI ALLE CONSORELLE: L'UBBIDIENZA}

Nel quarto sermone, dedicato all'ubbidienza, la clarissa veneziana morente riprende e illustra un altro dei capisaldi della vita monastica. Non mi dilungherò nella illustrazione della importanza di questa virtù, a cui Chiara Bugni dedica diverse pagine. Mi limiterò a sottolineare che la mistica ribadisce a più riprese la necessità

${ }^{56}$ Ibidem, f. 158r, p. 268.

${ }^{57}$ Ibidem, f. 166r, p. 274.

${ }^{58}$ Ibidem, f. 169v, p. 276.

${ }^{59}$ Ibidem, ff. 170r-v, pp. 276-277. 
che l'ubbidienza sia volontaria. E' infatti la rinuncia al libero arbitrio e la soggezione totale alle superiore e alle ufficiali del convento che rendono virtuosa la vita monastica. Questa rinuncia fa sì che l'ubbidienza possa essere definita un martirio senza spargimento di sangue ${ }^{60}$.

Dalla esortazione sull'ubbidienza ricaviamo il complesso semantico dei termini che accostano la professione monastica alla vita apostolica e cioè a quell'insieme di concetti e pratiche che possono suggerire la eventuale partecipazione delle donne al munus sacerdotale. Fin dall'esordio la Madre venerata dalle consorelle afferma solennemente che il suo ufficio comporta la "cura pastorale" delle monache e a questo ufficio elle vuole rimanere fedele fino alla morte:

Et però, havendo io a satisfar all'uficio della cura pastorale, spesso vi soglio ammonire all'osservanza della disciplina regolare, né mai da qui indietro vi mancherà la parola salutifera della salute per fin che lo spirito mio viverà et persevererà nella prigione di questa carne ${ }^{61}$.

La monaca viene definita da Chiara Bugni "soldata di Christo", secondo la metafora che paragona la vita spirituale ad un combattimento. Nei testi dell'epoca la figura paolina della milizia viene usata più spesso per designare l'identità maschile del monaco, essendo più consona alle donne l'immagine della sponsa Christi ${ }^{62}$. Tra le monache, l'abbadessa riveste il ruolo particolare di vicaria di Christo nel monasterio $^{63}$, o addirittura di vicaria di Dio $^{64}$ ed è per questo che la virtù dell'ubbidienza conduce alla perfezione, in quanto espressione della volontà di Dio mediata attraverso i superiori ecclesiastici. E' interessante notare che nell'ordinamento monastico che regola la convivenza di donne professe l'abbadessa è designata come vicario di Cristo allo stesso modo dei sacerdoti. In questo caso, il ruolo della monaca non pare avere bisogno di mediazioni maschili.

In qualità di vicaria di Cristo nel monastero, l'abbadessa deve sempre essere ubbidita, indipendentemente dalla sua bontà o miseria; l'ubbidienza le è dovuta da tutte le suore non per il suo merito ma per il nome di Cristo. Le consorelle debbono però prestare particolare attenzione ai suoi ammaestramenti:

La sua dottrina ti sia la regola del vivere et la sua santa conversatione ti sia esempio d'un ammaestramento molto puro. Perché ell'è amica di Dio et vicaria di Christo in terra; nel primo merita che tu l'ami, nel secondo che tu l'obbedisca in tutte quelle cose ch'essa ti comanda ${ }^{65}$.

Se poi accadrà alle monache di avere un'abbadessa rozza, ignorante o non troppo idonea, la professa stia attenta a non disseminare quello che lei fa $o$ che dice, ma pensi che i suoi peccati meritano che ella abbia una badessa disutile $^{66}$.

${ }^{60}$ Ibidem, f. 182r, p. 285.

${ }^{61}$ Ibidem, f. 177r, p. 282.

${ }^{62}$ Sull'identità di genere dei religiosi, mi permetto di rinviare a: G. Zarri, Religious Institutions, pp. 193-212; eadem, La vita religiosa, pp. 103-151. In prospettiva diversa $c f$. anche A.J. Schutte, Between Venice and Rome.

${ }^{63}$ Libro, f. 179v, p. 284.

${ }^{64}$ Ibidem, f. 177v, p. 282.

${ }^{65}$ Ibidem, ff. 179v-180r, p. 284.

${ }^{66}$ Ibidem, f. 179r, p. 284. 
Vediamo dunque quali sono i requisiti e le competenze dell'abbadessa, la figura femminile più prossima al sacerdote e al vescovo. Requisiti prioritari sono l'anzianità nella professione e la conoscenza della Sacra Scrittura, la mansuetudine e la modestia, la capacità di giudicare e di usare misericordia:

Ma ci sia una dona vecchia di costumi, erudita nelle scritture divine, che camini con lo spirito nella vera conversatione della santità, pia, mansueta, tranquilla di mente et discreta, la qual sappia giudicare le cattive e, a quelle che hanno buon volere usar miseratione ${ }^{67}$.

La fedeltà al voto fatto dalle monache al momento della professione è garanzia di perfezione e di salvezza. I fedeli cristiani si possono salvare vivendo rettamente secondo il Vangelo, ma le religiose non si salveranno se non osserveranno fino alla morte la regola del proprio ordine. Tramite l'ubbidienza alla abbadessa la monaca potrà camminare sicuramente e se persevererà insino alla fine combattendo et vincendo, gloriosamente sarà coronata ${ }^{68}$.

L'esortazione alla virtù della ubbidienza si accompagna all'invito di compiere un esame di coscienza circa l'osservanza dei voti e si conclude con un elogio per l'attenzione con cui le monache del Santo Sepolcro hanno seguito l'insegnamento della loro madre morente. Infine la considerazione della stanchezza delle consorelle spinge Chiara Bugni ad interrompere il sermone. Ella pensa che le compagne hanno assistito a una predica molto lunga e le ringrazia per averla ascoltata in così profondo silenzio che non s'è udita pur una di voi sputare, né forbirsi il naso ${ }^{69}$; conclude infine richiamando uno dei tratti propri della disciplina monastica: la meditazione della parola di Dio:

Vostro uficio sarà, come nell'altro sermone vi dissi, fare come il bue, ruminare quelle cose dette di sopra e pensarle molto bene, perché m'è parso avervi detto tutto quello che fa a proposito dell'ubbidienza ${ }^{70}$.

Andrà notato che nelle parole di Chiara Bugni citate in questo sermone ricorrono due termini propri della tradizione monastica: la conversatione, il cui ambito semantico si estende dalla parola detta in comunità alla vita vissuta insieme, e la ruminatio della parola di Dio, che bene esprime la forma e lo scopo della meditazione della Sacra Scrittura: un ripensamento individuale e interno della parola di Dio che viene mangiata e si trasforma in vita.

\section{IL CONGEDO DI CHIARA BUGNI: LA PREDICAZIONE FEMMINILE}

I primi sermoni dettati alle compagne da Chiara Bugni erano indirizzati alla riflessione sulla disciplina monastica e sul modo di perseguire la salvezza individuale. Trascorsi tre giorni dall'inizio della predicazione, le consorelle si avvicinano ancora all'abbadessa ammalata e le chiedono una riflessione sulla morte. Chiara non lo nega, sapendo che presto lascerà coloro che le sono state sottoposte e hanno condiviso con lei la vita comune. Il sermone sulla morte è un'occasione per parlare della resurrezio-

${ }^{67}$ Ibidem, f. 178 r, pp. 282-283.

${ }^{68}$ Ibidem, f. 184 r, p. 287.

${ }^{69}$ Ibidem, f. 185 r, p. 288.

${ }^{70}$ Ibidem, f. 185 v, p. 288. 
ne di Cristo e del corpo e per prendere definitivo congedo dalle sorelle che assistono al conferimento del viatico e della estrema unzione alla Madre morente.

E' proprio in quest'ultimo discorso che viene toccato il problema della predicazione femminile nell' ambito dei doveri abbaziali. Qui si esplicita compiutamente il senso del sermone come conversatio tra fratelli, poiché le monache intessono con l'abbadessa un dialogo intenso. Il discorso inizia con una perorazione di Chiara sulla morte, che si presenta letterariamente molto bella ed è ricca di citazioni indirette della Sacra Scrittura:

Ecco ch'io mi spoglio questo vestimento ch'è mortale, acciò che io me ne vesta d'uno immortale. Per insino a hora io sono stata in peregrinaggio, horamai io ritorno alla mia patria. Ecco che io piglio il palio per il quale ho combattuto nel monasterio. Ecco che io tocco il porto, il quale con tanto desiderio io ho desiderato ${ }^{71}$.

A queste parole rispondono le sorelle, piangendo:

Chi haveremo più noi che ci consoli, ci ammaestri et ci insegni? (...). Voi eravate la nostra cara madre, la nostra maestra, il nostro refugio, la nostra consolatione et un essempio d'innocentia (...). Horamai verremo a niente, saremo come pecore senza pastore (...). Non sarà chi ci consoli, rimarranno orfane le vostre figliuole et patiranno la fame come cani, andandovi cercando per tutto e non vi trovando ${ }^{72}$.

Di rimando l'abbadessa consola le consorelle e ricorda loro la storia del popolo d'Israele. Dopo la morte di Mosè, Dio elesse Giosuè "per guida e diffensore al populo suo" e avendo portato Elia in cielo con un carro di fuoco lasciò al suo popolo Eliseo come profeta sopra lo quale si riposò lo spirito doppio di Elia ${ }^{73}$. Come ha fatto con Israele, il Signore invierà anche alle monache veneziane qualcuno in grado di prendere il posto della mistica visionaria e che avrà i requisiti dell'abbadessa santa:

Il Signore sarà la parte della vostra heredità et del vostro calice, et esso vi restituirà la vostra heredità et vi susciterà un'altra Madre, alla quale darà il verbo di predicarvi il vangelo; et essa camminerà senza macchia et opererà la giustitia ${ }^{74}$.

Prima di congedarsi dalle compagne di vita, Chiara Bugni cerca di frenare la commozione del momento e il dolore del distacco ricorrendo alla consolante realtà della istituzione monastica, che garantisce la continuità degli uffici e la trasmissione dei carismi. Ogni abbadessa, e non soltanto colei che gode fama di santità, ha il compito di insegnare e predicare alle monache nel contesto della quotidiana conversatio.

Nel passo citato appare pienamente chiarito il senso della predicazione femminile all'interno delle comunità regolari: un insegnamento "in forma di sermone", condotto da coloro che esercitano un ufficio al servizio delle sorelle, rivolto in primo luogo alle monache, negli spazi comuni del monastero, quando non addirittura al letto di morte dell'abbadessa.

${ }^{71}$ Ibidem, f. 194r, p. 294.

${ }^{72}$ Ibidem, f. 194v, p. 295.

${ }^{73}$ Ibidem, f. 195v, p. 295.

${ }^{74}$ Ibidem, f. 195v, p. 296. 
In conclusione, la Vita e le Esortazioni della clarissa Chiara Bugni possono essere considerate a mio parere un caso esemplare per illustrare il doppio binario della predicazione femminile: quella profetica, affidata alle rivelazioni e alle visioni, rivolta al pubblico dei fedeli e per lo più mediata da un confessore che ne garantisce l'autenticità e l'ortodossia; e quella "in forma di sermone", riservata all'insegnamento interno alla comunità e trasmessa ex officio alle monache nell' ambito della "conversazione" monastica.

\section{BIBLIOGRAFIA}

Arcangeli, Letizia, Ragioni politiche della disciplina monastica: Il caso di Parma tra '400 e '500, in Zarri, Gabriella (ed.), Donna, disciplina, creanza cristiana dal XV al XVII secolo. Studi e testi a stampa, Roma, Edizioni ai Storia e Letteratura, 1996, pp. 165-187.

Barone, Giulia; Dalarun, Jacques (eds.), Angèle de Foligno. Le dossier, Roma, École Française de Rome, 1999.

Busi, Giulio, Francesco Zorzi. Un metodico sognatore, in Idem, L'enigma dell'ebraico nel Rinascimento, Torino, Aragno, 2007, pp. 161-186.

Cantimori, Delio, Le idee religiose del Cinquecento. La storiografia, in Cecchi, Emilio; Sapegno, Natalino (eds.), Storia della letteratura italiana. Il Seicento, Milano, Garzanti, 1967, pp. 7-53.

Caterina Vigri, I Sermoni, introduzione e commento di Gilberto Sgarbi con un saggio di Enzo Lodi, Bologna, Barghigiani, 1999.

Caterina Vigri, Laudi, trattati e lettere. Edizione critica a cura di Silvia Serventi, Firenze, Sismel, 2000.

Cavalli, Stefania, Beata Chiara Bugni (1471-1514), monaca del monastero del Santo Sepolcro a Venezia. Edizione critica della vita, tesi di Laurea Università di Venezia, anno accademico 2000-2001, relatore prof. Reinhold Mueller.

Jansen, Katherine L., The Making of the Magdalen. Preaching and Popular Devotion in the Later Middle Ages, Princeton, New Jersey, Princeton University Press, 1999.

Kay McNamara, J.A., Sisters in Arms. Catholic Nuns through Two Millennia, Cambridge - London, Harvard University Press, 1996.

Kienzle, Beverly M.; Walker, Pamela J. (eds.), Women Preachers and Prophets throught Two Millennia of Christianity, Berkeley - Los Angeles - London, University of California Press, 1998.

Leonardi, Claudio (ed.), Caterina Vigri: la santa e la città : atti del Convegno, Bologna, 13-15 novembre 2002, Firenze, Sismel Edizioni del Galluzzo, 2004.

Lowe, Kate J.P., Nuns' Chronicles and Convent Culture in Renaissance and CounterReformation Italy, Cambridge, Cambridge University Press, 2003.

Marco da Lisbona, Croniche de li Ordini instituiti dal P. san Francesco, parte IV, vol. III, raccolto principalmente dal M.R.P. Bartolomeo Cimarelli, Napoli, Novello De Bonis, 1680.

Matter, E. Ann; Zarri, Gabriella, Una mistica contestata. La Vita di Lucia da Narni (1476-1544) tra agiografia e autobiografia. Con l'edizione del testo, Roma, Edizioni di Storia e Letteratura, 2011.

Mayne Kienzle, Beverly; Walker Berkeley, Pamela (eds.), Women Preachers and Prophets throught Two Millennia of Christianity, Los Angeles - London, University of California Press, 1998.

McNamara, Jo Ann K., Sisters in Arms. Catholic Nuns through Two Millennia, Cambridge - London, 1996. 
Mueller, Reinhold C.; Zarri, Gabriella (eds.), La Vita e $i$ Sermoni di Chiara Bugni clarissa veneziana (1471-1514), Roma, Edizioni di Storia e Letteratura, 2011.

Mueller, Reinhold C., Ambienti ecclesiastici e laici attorno alla figura di Chiara Bugni, in Mueller, Reinhold C.; Zarri, Gabriella (eds.), La Vita $e$ i Sermoni di Chiara Bugni, Roma, Edizioni di Storia e Letteratura, 2011, pp. 63-122.

Nicolini, Ugolino (ed.), Memoriale di Monteluce: cronaca del monastero delle clarisse di Perugia dal 1448 al 1838, Santa Maria degli Angeli (Assisi), Porziuncola, 1983.

Rauch, Simone, La Vita della beata Chiara Bugni: formazione e tradizione dei testimoni, in Mueller, Reinhold C.; Zarri, Gabriella (eds.), La Vita $e$ i Sermoni di Chiara Bugni, Roma, Edizioni di Storia e Letteratura, 2011, pp. 3-26.

Ricordanze del Monastero di Santa Lucia OSC in Foligno: cronache 1424-1786. Introduzione, trascrizione, note, indici a cura di Sr. A. Emmanuela Scandella OSC; appendice su altri monasteri OSC in Umbria a cura di P. Giovanni Boccali OFM, Assisi, Porziuncola, 1987.

Roest, Bert, Predicazione femminile nel tardo medioevo tradizione francescana, "Studi Francescani" 62 (2004), pp. 119-154.

Sanudo, Marin, I Diarii, Venezia, Tipografia del commercio di Marco Visentini, 1879. 1903, vol. VII.

Schutte, Jacobson, Anne, Between Venice and Rome: the Dilemma of Involuntary Nuns, Kirksville, Truman State University, 2010.

Vasoli, Cesare, Intorno a Francesco Giorgio Veneto e all'Armonia del mondo, in Vasoli, Cesare, Profezia e Ragione. Studi sulla cultura del Cinquecento e del Seicento, Napoli, Guida, 1979, pp. 129-403.

Vasoli, Cesare, Un "precedente" della Vergine Veneziana. Francesco Giorgio Veneto e la clarissa Chiara Bugni, in Leather Kuntz, Marion (ed.), Postello, Venezia e il suo Mondo, Firenze, Olschki, 1988, pp. 203-225.

Vasoli, Cesare, Nuovi documenti sulla condanna all'indice e la censura delle opere di Francesco Giorgio Veneto, in Stango, Cristina (ed.), Censura ecclesiastica e cultura politica in Italia tra Cinquecento e Seicento, Sesta giornata Luigi Firpo: atti del Convegno, 5 marzo 1999, Firenze, Olschki, 2001, pp. 55-78.

Wadding, Luca, Annales minorum seu trium ordinum a S. Francisco institutorum, Romae, Ad Claras Aquas (Quaracchi), 1931, vol. VII, pp. 541-548.

Walker Bynum, Caroline, Wonderful blood: theology and practice in late medieval northern Germany and beyond, Philadelphia, University of Pennsylvania, 2007.

Zarri, Gabriella, Le sante vive. Cultura e religiosità femminile nella prima età moderna, Torino, Rosenberg \& Sellier, 1990.

Zarri, Gabriella, Religious Institutions and Social Discipline: The Reform of the Regulars, in Brown, Judith C.; Davis, Robert C. (eds.), Gender and Society in Renaissance Italy, Essex, Addison Wesley Longman, 1998, pp. 193-212.

Zarri, Gabriella, La vita religiosa tra rinascimento e controriforma. Sponsa Christi: nozze mistiche e professione monastica, in Mattews Greco, Sara; Brevaglieri, Sabina (eds.), Monaca moglie serva e cortigiana. Vita e immagine delle donne tra rinascimento e controriforma, Firenze, Morgana Editrice, 2001, pp. 103-151.

Zarri, Gabriella; Baranda Leturio, N. (eds.), Memoria e comunità femminili: Spagna e Italia, secc. XV-XVII - Memoria y comunidades femmininas: España e Italia, siglos XV-XVII, Firenze, Firenze University Press - UNED, 2011.

Zarri, Gabriella; Chiacchella, Rita (coords.), Memoriale di Monteluce. Cronaca del Monastero delle clarisse di Perugia dal 1839 al 1927, vol. II, Santa Maria degli Angeli, Assisi, Porziuncola, 2003. 
Zarri, Gabriella, Predicatrici e madri spirituali. Il carisma, lo spazio, il pubblico, in Corsi, Dinora (ed.), Donne cristiane e sacerdozio. Dalle origini all'età contemporanea, Roma, Viella, 2004, pp. 159-177.

Zarri, Gabriella, Venetian Convents and Civic Ritual, in Weaver, Elissa B. (eds.), Arcangela Tarabotti. A literary Nun in Baroque Venice, Ravenna, Longo Editore, 2006, pp. 37-56.

Zarri, Gabriella, Madri dell'anima: Chiara Bugni, Elena Duglioli e la rigenerazione della chiesa, "Micrologus. Natura, Scienze e Società Medievali" 27 (2009), pp. 415-435.

Zarri, Gabriella, Places and Gestures of Women's Preaching in Quattro-and Cinquecento Italy, in Jansen, Katherine L.; Rubin, Miri (eds.), Charisma and Religious Authority. Jewish, Christian, and Muslim Preaching 1200-1500, Turnhout, Brepols, 2010, pp. 177-193.

Zarri, Gabriella, Chiara Bugni e Francesco Zorzi suo biografo: saggio introduttivo, in Mueller, Reinhold C.; Zarri, Gabriella (eds.), La Vita $e$ i Sermoni di Chiara Bugni, Roma, Edizioni di Storia e Letteratura, 2011, pp. 11-39.

Zorzi, Francesco, L'armonia del mondo. Testo latino a fronte. Saggio introduttivo, traduzione, note e apparati di Saverio Campanini, Milano, Bompiani, 2010, pp. 11-43.

Fecha de recepción del artículo: enero 2012

Fecha de aceptación y versión final: marzo 2012 